\title{
ALIGNING GOAL AND PROCESS MODELS
}

\author{
Arvind Kumar ${ }^{1}$, Suhas Pande ${ }^{2}$ \\ ${ }^{I}$ Manager, Business Transformation, IT Strategies, Derex Technologies \\ ${ }^{2}$ Solution Architect (IT and Security), Derex Technologies
}

\begin{abstract}
Business Process Modeling (BPM) notations such as BPMN are used to effectively conceptualize and communicate important process characteristics to relevant stakeholders. BPMN gives an overview of the entire process of the particular organization but does not contain the notion of business goals and non-functional requirements. On the other hand Agent-Oriented conceptual modeling (AOCM) notations like $i *$ are highly effective in representing requirements from an intentional stance and answering questions such as what goals exist, how key actors depend on each other and what alternatives must be considered but does not have the process flow. In this paper we illustrate, through a case study a methodology that would help all stakeholders to identify, at multiple levels of abstraction, the goals and their corresponding activities in a business environment.
\end{abstract}

Keywords: BPMN, Goals, Requirements Modelling.

\section{INTRODUCTION}

A BPM system needs to provide a representation of the process of an organization which is meaningful to a business person. Many organizations use BPM to not only model their existing state but also use it as a requirements gathering exercise. These organizations have business analysts who work with the business partners to model the business process flow. Similar to the concept of using UML (Rumbaugh et al. 2004) use cases and business rules requirements in form of rules repository, the analysts capture business process flow in form of process description and BPMN (OMG 2011) model. However, BPMN 2.0 has many shortcomings some which have been clearly described in (Borger 2012). The central argument against using regular BPMN is that resource management can be expressed only via lanes (actors, roles) or performers of user or manual tasks. No execution time parameters, goals or softgoals are considered. To be effective, a business process should aim at a goal. However, BPMN only includes a series of structured activities that transform inputs into outputs bringing some value to a customer or to the market. Thus, BPMN alone cannot be fully used to model the entire set of requirements and must be augmented by other richer notations. Current business process modeling notations do not model quality information such as information about usability or time behavior of a business process.

Agent-oriented conceptual modeling (AOCM) notations such as $i^{*}(\mathrm{Yu}$ 1997) represents an interesting approach for modeling early phase requirements which includes organizational contexts, stakeholder intentions and rationale. Although both the fields of Goal-Oriented RE (GORE) and BPM received a lot of attention from researchers, the methods to transform goal models into business process models still need further research (Decreus et al. 2009). Goal-oriented process behavior can be seen as an internal guide to a process that helps to clarify why we do the process and verify if we achieve it. $i *$ incorporates the goals and the non-functional requirements of the system but does not have the process flow.
Since the active participation of all stakeholders is a key factor for successful requirements engineering, it is important that BPM be shared by all stakeholders. However, organizations often end up with inconsistent BPM which do not cover all stakeholders' needs and constraints. Thus there is a need for a modeling notation that would be meaningful to both the software developer as well as the business stakeholder (which also includes the end-users). The diagram must not be cluttered by aspects which are implementation oriented as opposed to business oriented.

In this paper, we illustrate the use of a methodology that we feel would facilitate and support the combined use of modeling business processes and goal-oriented requirement engineering process in a synergistic fashion. We do a case study and illustrate how our methodology can be used to model both the business process and goals. We also talk briefly about a tool that we are currently building that would enable a system analyst to use our proposed methodology.

The remainder of this article is organized as follows. Section 2 talks about our research objective and Section 3 gives a brief background of BPMN and the $i^{*}$ framework. Section 4 discusses our proposed methodology and gives an overview of a tool (BPGM) that we are currently building to support our work. Section 5 describes a case study where we applied our methodology. Section 6 discusses related work and the concluding section talks about future work.

\section{RESEARCH OBJECTIVE}

Researchers have tried to reason the correspondence between goal models and business process models and offer guidelines in terms of when to use one or the other or both (Vara et al. 2013). From a notational diagram perspective, having the best of both worlds ( $i^{*}$ and BPMN) for the purpose of modelling the system would be the best where all stakeholders as well as the business analyst would get an overall view of the entire system in a comprehensive manner. But even when we join the two for a better understanding of the system for all its stakeholders, the 
resulting diagram may be not that easy to comprehend. One has to think that the understanding should take into consideration the target audience of the artefact and not always something that offers lots of information helps. There has been several business process modeling notations over the years as discussed (Monsalve et al. 2010) however, till date there is not one notation to satisfy all.

Given that there are multiple points of view of looking at a business process, our research objective is to find out a methodology in which the co-evolution of both $i^{*}$ and BPMN diagrams would be possible. Furthermore, our aim is to create a tool in which would help the analyst to capture both the models and translation from one model to another would be possible. This would enable all parties concerned the business stakeholders, the end-users and the system analyst to get a consistent view of the current business activities and organizational and user goals and softgoals

\section{BACKGROUND}

\subsection{BPMN}

Business Process Modeling Notation (BPMN) (OMG 2011), developed by the Business Process Management Initiative (BPMI.org) and controlled by the Object Management Group(OMG) is based in the representation of activities flows that consider the resource, decision making and events. The current specification document (BPMN 2.0) states that the objective of the standard is for providing a notation that would be understood well by all stakeholders. The objective clearly indicates that BPMN comprises two parts: a business view and a technical view. The business view targets the business analysts who will use BPMN to create descriptive business processes models which can be communicated and analyzed. The technical view targets technical developers who will need to add detailed technical specifications to the models to make them executable. The separation between the two parts in BPMN is in line with the separation between the domain-oriented components and design-oriented components in a conceptual model. Thus business users who are more interested in the domainoriented components can use the business view part of BPMN. Likewise, the design oriented components which require detailed specifications of the model can be represented using the technical part of BPMN.

Figure 1 presents the organization in terms of Pool and lanes, inside these elements there are the sequence of activities that are executed in the process rep- resented by Tasks, Flow links, and Sub-Process. The events represent the triggers and stop events of process, such as, Start, Stop and intermediate events. The resources produced or consumed by the process are represented as Artifacts that could be input and output of tasks and sub-processes. Moreover, the communication between different organizations (pools) and people (lanes) is performed by connections of Message.

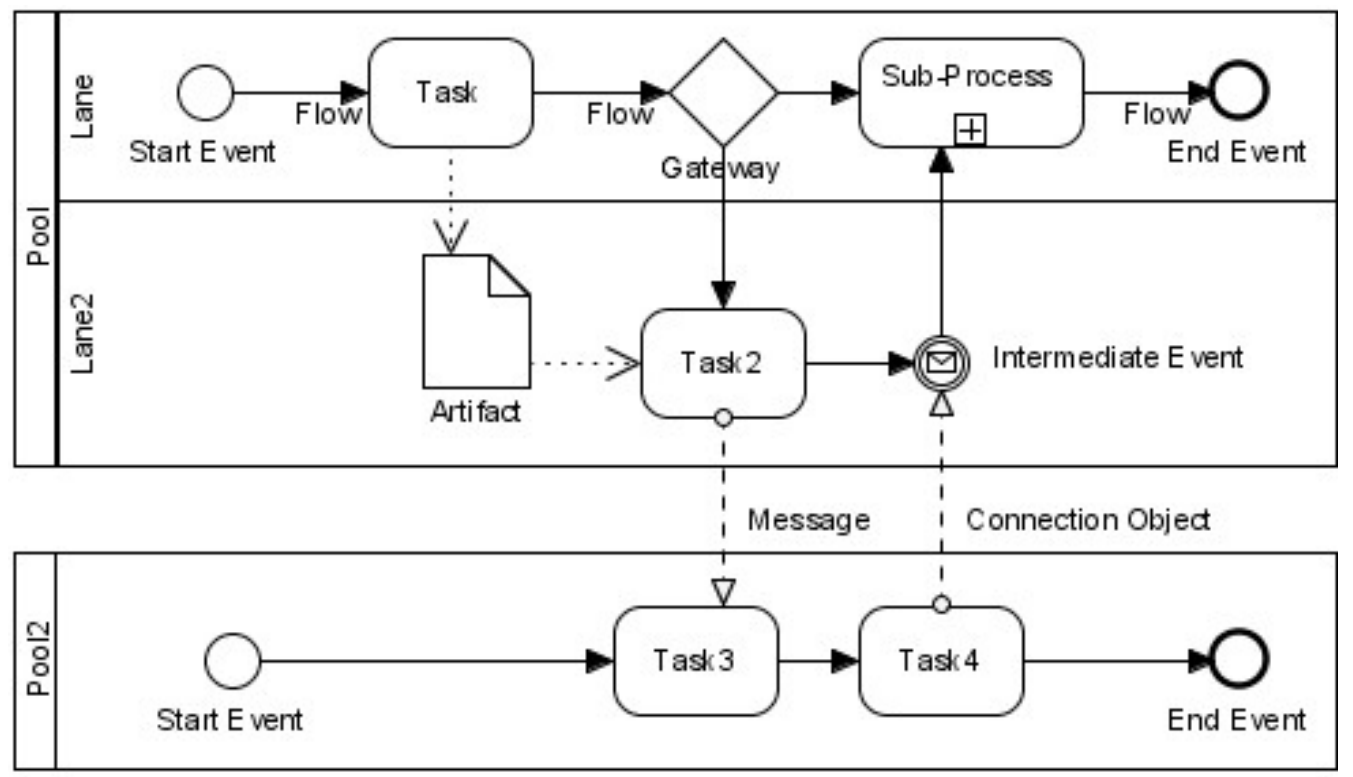

Fig 1: Sample BPMN diagram

\subsection{The i* Framework}

$i^{*}(Y u$ 1997) is an agent-oriented modeling framework that can be used for requirements engineering, business process reengineering, organizational impact analysis, and software process modeling. $i^{*}$ supports the modeling activities that take place before the system requirements are formulated, it can be used for both the early and late phases of the requirements engineering process. During the early requirements phase, the $i^{*}$ framework is used to model the environment of the system-to-be. It facilitates the analysis of the domain by allowing the modeler to diagrammatically represent the stakeholders of the system, their objectives, and their relationships. An $i^{*}$ model consists of two main modeling components: the Strategic Dependency (SD) Model and the Strategic Rationale (SR) Model. 
The SD diagram consists of a set of nodes and links. Each node represents an "actor", and each link between the two actors indicates that one actor depends on the other for something in order that the former may attain some goal. The depending actor is known as depender, while the actor depended upon is known as the dependee. The SR diagram represents the goals, task, resource, and soft- goal dependencies between actors. There are four types of dependencies in $i^{*}$ - goal dependency, task dependency, resource dependency and softgoal dependency. The notion of softgoals (quality goals) is related to the notion of non- functional requirements [5]. Softgoals are the goals that do not have a clear-cut satisfaction condition. Each contribution link towards a softgoal is characterized by a label that specifies the contribution type and strength. The positive contribution types for softgoals are HELP (positive but not by itself sufficient to meet the higher goal), MAKE (positive \& sufficient), SOME+ (partial positive contribution), and '?' (unknown). The dual negative types are HURT, BREAK, and SOME- respectively. Sample SD and SR model of a Meeting Scheduler are shown in figures 2 and 3 which has been taken from [19].

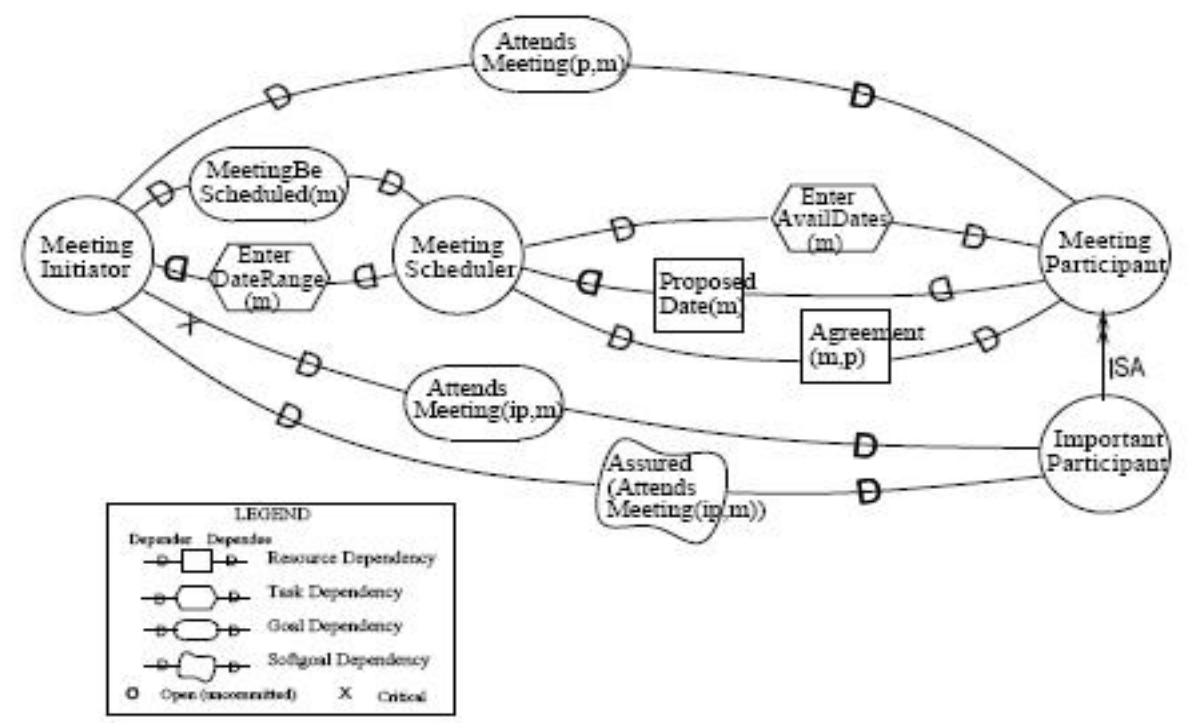

Fig. 2. SD diagram Meeting Scheduler
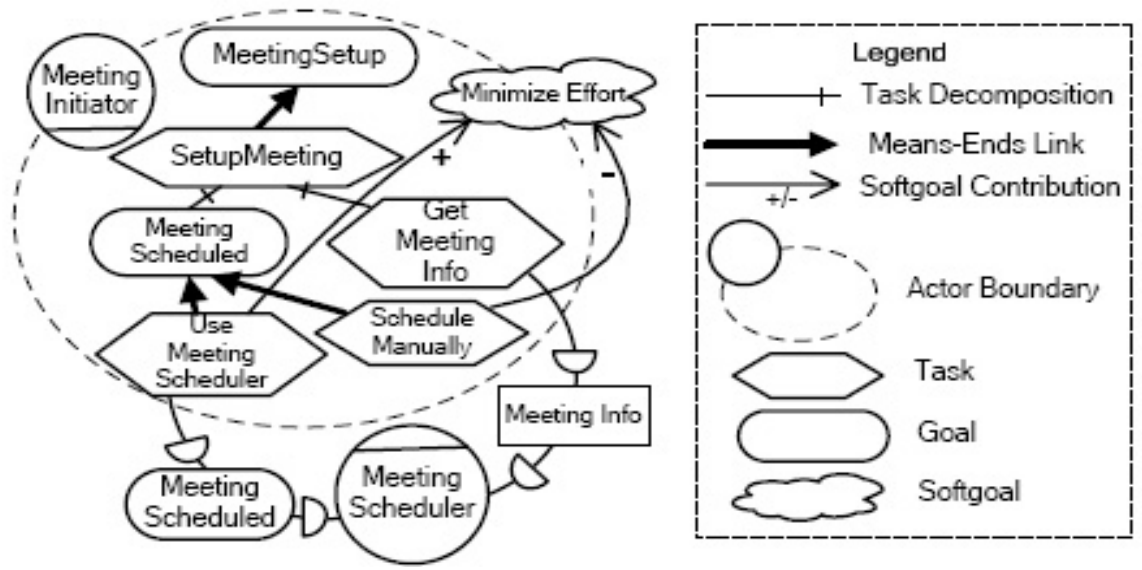

Fig. 3. SR diagram for Meeting Initiator Agent

\section{OUR PROPOSED METHODOLOGY}

As stated earlier, our goal is to create a methodology through which both workflow and goal models can be analysed. One of the key aspects of defining the methodology is to first have a process model which captures all relevant information as outlined below:

- Sequence Flow

- Roles
- Activities

- Events

- Process Hierarchies

- Organizational Hierarchy

- Goal modeling

- Goal model Evaluation

- Goal/Process Traceability

- Softgoal modeling

- Operationalization of softgoals 
Unfortunately none of the notations as discussed earlier support all these activities Moreover, by aligning business goals and softgoals to process models in a traceable way would greatly contribute goal satisfaction and process adaptation. In order to get all the above relevant information from which software requirements can be generated we propose the following 6 steps:

1. Understand the business process from existing documents and interacting with the end users of the business process and generate a BPMN diagram (if not already available).

2. Extract goal model (i* diagram) from the existing BPMN diagram from each actors' perspective using an approach described in (Vara et al. 2013).

3. Conduct interviews with business stakeholders and users to understand the entire organizational as well process goals/softgoals and creation of $i^{*}$ diagram.

4. Align the goal models derived from steps 2 and 3 and check if they are consistent. There is no specific methodology to check the two models. Particularly, softgoals cannot be obtained from BPMN diagrams (in step 2) and organizational goals (obtained in step 3) may have to be decomposed using a goal hierarchy into subgoals.

5. Re-design the process model and update the BPMN and $i^{*}$ diagrams in case conflicts are found in step 4. This may require several iterations from steps 1 to 4 before a final design may be arrived at. It would also mean revisiting the original BPMN diagram to include subprocesses in greater detail which may otherwise have not been documented in original BPMN (goal operationalization).

6. Operationalize softgoals with tasks for each individual actor since softgoals cannot be directly shown in a BPMN diagram. The process requires talking to the end users as well as the management so that priorities may be defined. It is to be noted that there may be conflicting softgoals which nevertheless must be operationalized so that they can be attributed to some tasks/processes.

7. Generate a requirement specification document from the above steps.

\subsection{Business and Goal Modeling Tool - BPGM}

We are working on creating a tool called BPGM which would help one to capture all the elements discussed above as well as help us to create a model that would have both the business process model view as well as the goal model view. We believe that a tool of this kind help an organization to align the business objectives along with the process activities. The tool would store the model in a database from where extraction of relevant data from each stakeholder's point of view could be obtained. Some of the features of the tool would be as follows:
- The tool would take as input the BPMN and i* models and would have the ability to generate one from the other.

- The tool would help to zoom in and zoom out on particular activities thereby giving a glimpse of all subprocesses within a particular process. Related goals and softgoals can also be viewed at all levels.

- The tool would provide a detailed methodology for modifying (addition, deletion, substitution) any part of the system. As stated in (Lapouchnian 2005) the modification methodology for goal models has not been considered in detail by researchers.

\section{CASE STUDY}

A case study was conducted on an engineering services company in India, which specializes in carrying out entire projects. Their work consists of taking up the entire responsibilities of surveying, studying operational requirements, preparing of schemes, getting approval from appropriate authorities, procuring of equipment, erecting, testing and commissioning including carrying out related civil and structural works etc. The company mostly works with government organizations and gets projects based on the government invited tenders that they win. One of the main focuses of the organization is therefore on submitting strong tenders. The company has a clearly defined workflow model for the tendering process that is mostly done manually. The company has been doing this process for several years now and the knowledge gained from a tender application (whether successful or failed) is not really documented or kept in an easily retrievable format (like reports) from which future tender applications may get benefitted. The company therefore needed IT enablement of some of its processes to not only keeps the records but also to have the knowledge embedded inside the company instead of some individual employees.

The participant company was interested in modeling the business processes in order to: 1) document them; 2) automate part of the system; 3) communicate to their new employees the Tendering Process functional characteristics and 4) create a knowledge repository. The case study was planned for 3 months of work, and it required the participation of various members of the company - the individual users who performed the various activities as well as the owner and top-executive of the company who could give not only a technical perspective but also commercial and organizational perspectives of the business processes. It is to be mentioned here that none of them had previous experience with BPM or goal models.

Based on the interaction with the personnel involved in the process of tender submission a BPMN diagram was prepared as shown in Figure 4 which is the first step in our methodology described earlier. 


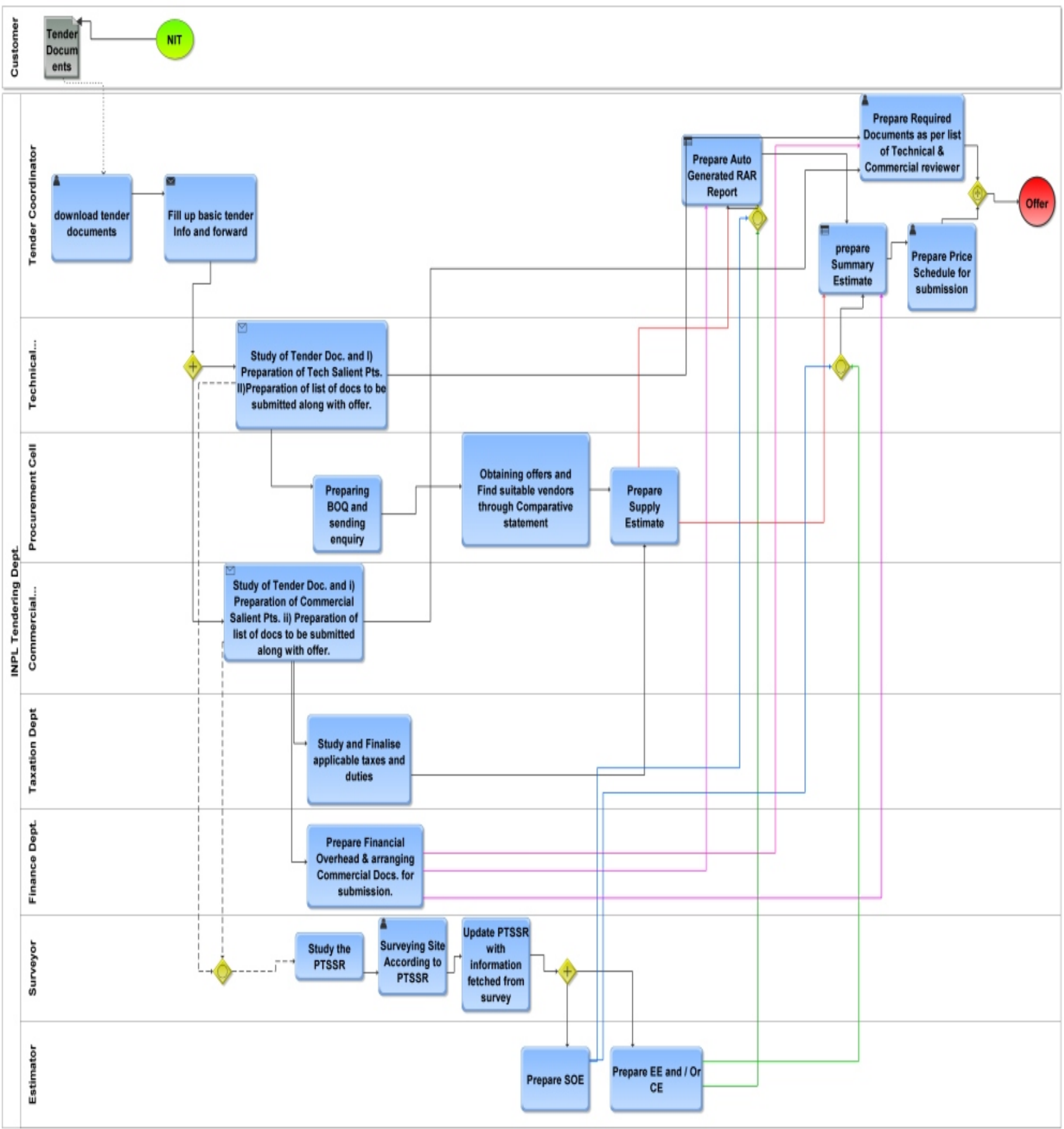

Fig. 4. Initial BPMN diagram for Tendering Process

We started work on the generation of $i^{*}$ model from the BPMN diagram in order to align the business process with goals. While this activity was a straight-forward one, once we started interviewing the various stakeholders we found that several goals and softgoals were not addressed in the activity. As an example, security aspects of the tendering process or knowledge management from earlier tenders were neither practiced nor depicted in the BPMN diagram though these were some of the top goals/softgoals of the organization. Figure 5 depicts portion of the modified $i^{*}$ SR diagram after step 4.

We also worked on the operationalization of softgoals and after several meetings with the stakeholders we were finally able to create both BPMN and $i^{*}$ models that would reflect an entire process model for the Tendering Process for the company. 


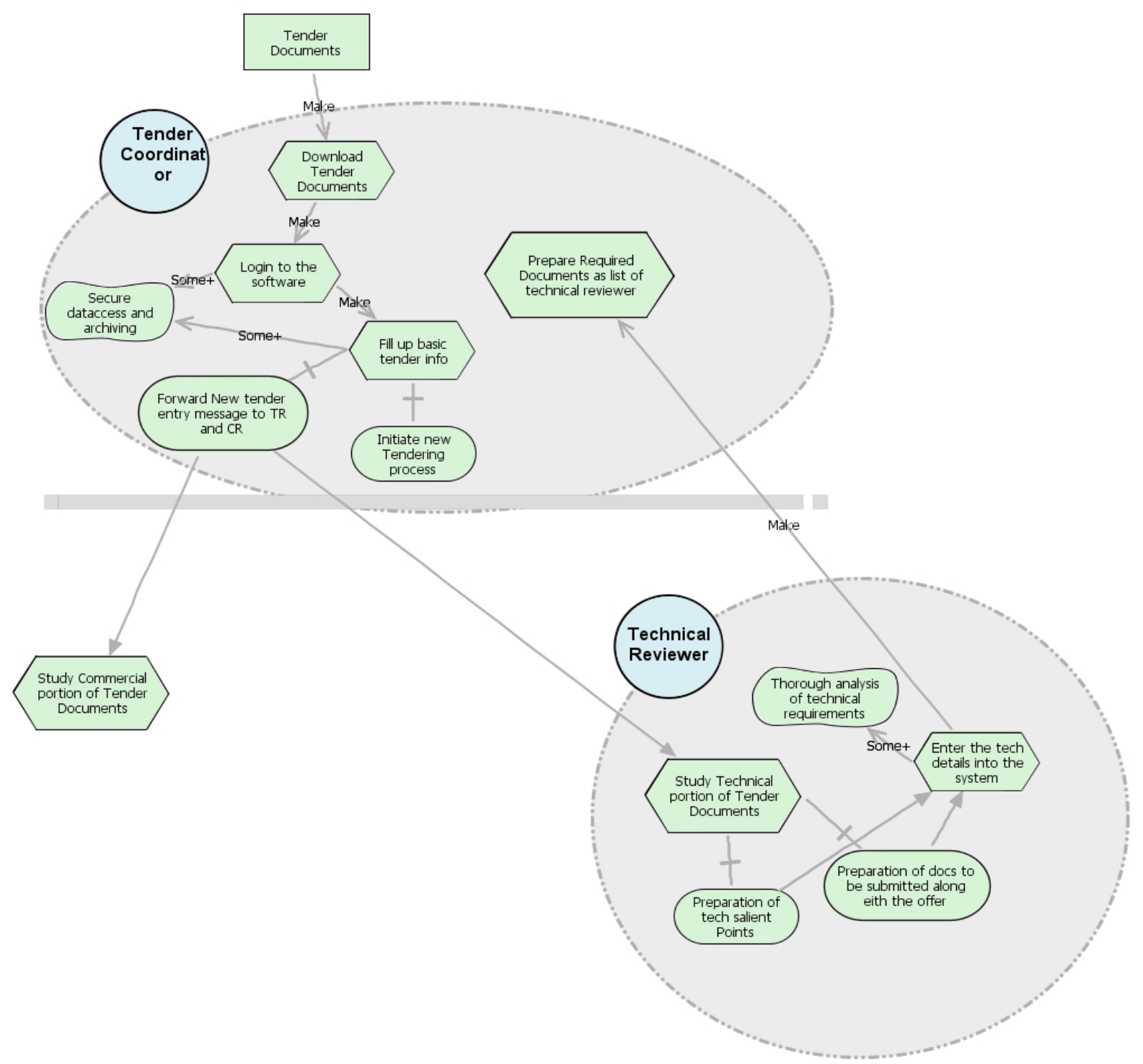

Fig. 5. Partial SR diagram for Tendering Process

\subsection{Results}

Our research was well appreciated by both the users as well as the management of the company who felt that the models were easy to understand and reflected the company's business objective. However, the biggest concern was the difficulty in viewing the diagrams in both digital and paper format because of their enormous size. Trying to depict the entire process through different notations also meant the users had to learn new concepts to understand the diagrams fully. In order to overcome the above limitations, we are now working on the BPGM tool which we believe would solve much of the existing problems.

\section{RELATED WORK}

There has been several research works relating to the combined modeling of BPMN and GORE which have tried to address the problem of incorporating Goal models into BPMN.
In (Pavlovski and Zou 2008), the authors assume that business process modeling is concerned about capturing functional behavior of business process but fail to cover non-functional requirements. To tackle this deficiency, the authors propose two new artifacts to capture these constraints denominated operating condition (to represent the business process constraints) and control case (to denote criteria to manage the risk associated with a given operational condition), to be applied in business process models. For that, it extends BPMN with these constructs. In (Alencar et al. 2009) the authors propose a transformation process to make it possible the transformation of the $i^{*}$ models into a preliminary conceptual model for the ObjectOriented Method approach with BPMN. Bhuiyan et. al. in (Bhuiyan and Krishna 2010) applied a method of coevolution of operational (BPMN) and organizational $\left(i^{*}\right)$ models in an industrial setting which provided a way of expressing changes, which arise in one model, effectively in the other model. They used constrained development methodologies (Koliadis et al. 2006) capable of guiding an analyst when reflecting changes from an $i^{*}$ model to a BPMN model and vice-versa. 


\section{CONCLUSION}

In this paper we have provided a methodology whereby coevolution of business process and goal models take place. Our work incorporates the process flow from Business Process Model on one hand and the goals, sub-goals and non-functional requirements from $i^{*}$ diagram on other hand. We have further illustrated the methodology by conducting a case study of a business process the results of which are quite encouraging to the stakeholders of the company as well as provide a requirements document for automating some of their processes.

We are currently developing a tool (BPGM) which would enable an analyst to carry out our methodology in an semiautomated manner. It would also help the organization stakeholders to get a better understanding of their processes. Beyond the basic tool we also plan to incorporate the following so as to enhance to quality of the model:

Optimization: Inclusion of the suitable algorithms in the software and using various concepts of graph theory to optimize business process flows.

Knowledge Management: Incorporation of knowledge management.

Norm Compliance: Adherence to norms and laws.

Priority Allocation: By allocating different priority values we can determine the most preferred model as mentioned in (Liaskos et al. 2010).

\section{REFERENCES}

[1] Alencar, F., Marn, B., Giachetti, G., Pastor, O., Castro, J., and Pimentel, J. (2009a): "From i* Requirements Models to Conceptual Models of a Model Driven Development Process". In: The Practice of Enterprise Modeling. Ed. by Anne Persson and Janis Stirna. Vol. 39. Lecture Notes in Business Information Processing. Springer Berlin Heidelberg, pp. 99-114. ISBN: 978-3642- 05351-1. DOI: 10.1007/978-3-642-05352-8 9. URL: http: //dx.doi.org/10.1007/978-3-642-05352-8 9.

[2] Bhuiyan, M. \& Krishna, A. (2010). Business modeling with the support of multiple notations in requirements engineering. $14^{\text {th }}$ Pacific Asia Conference on Information Systems, PACIS 2010 (pp. 1766-1773).

[3] Borger, E. (2012): "Approaches to modeling business processes: a critical analysis of BPMN, workflow patterns and YAWL". In: Software and System Modeling 11.3, pp. 305-318.

[4] Chung, L., Nixon, B. A., Yu, E., and Mylopoulos, J. (2000): Non-Functional Requirements in Software Engineering. Kluwer Academic Publishers.

[5] Decreus, K., Snoeck, M., and Poels, G. (2009): "Practical Challenges for Methods Transforming i* Goal Models into Business Process Models". In: Requirements Engineering Conference, 2009. RE '09. 17th IEEE International, pp. 15-23. DOI: 10.1109/RE.2009.25.

[6] Koliadis, G., Vranesevic, A., Bhuiyan, M., Krishna, A., and Ghose, A. K. (2006): "Combining i* and BPMN for Business Process Model Lifecycle Management". In: Business Process Management Workshops. Ed. by
Johann Eder and Schahram Dustdar. Vol. 4103. Lecture Notes in Computer Science. Springer, pp. 416-427. ISBN: 3-540-38444-8.

[7] Lapouchnian, Alexei (2005): "Goal-oriented requirements engineering: An overview of the current research". In: University of Toronto.

[8] Liaskos, S., McIlraith, S.A., Sohrabi, S., and Mylopoulos, J. (2010): "Integrating Preferences into Goal Models for Requirements Engineering". In: Requirements Engineering Conference (RE), 2010 18th IEEE International, pp. 135- 144. DOI: 10.1109/RE.2010.26.

[9] Monsalve, C., April, A., and Abran, A., "Representing Unique Stakeholder Perspectives in BPM Notations," Software Engineering Research, Management and Applications, ACIS International Conference on, pp. 42-49, 2010 Eighth ACIS International Conference on Software Engineering Research, Management and Applications, 2010

[10] OMG (2011): "Business Process Model and Notation (BPMN) Version 2.0”. In: URL: http://www.omg.org/spec/BPMN/2.0.

[11] Pavlovski, C.J. and Zou, J. (2008): "Non-functional Requirements in Business Process Modeling". In: Proceedings of the Fifth Asia-Pacific Conference on Conceptual Modelling - Volume 79. APCCM '08. Wollongong, NSW, Australia: Australian Computer Society, Inc., pp. 103-112. ISBN: 978-1-920682-60-6. URL: http://dl.acm.org/citation.cfm?id=1379429.1379443.

[12] Rumbaugh, J., Jacobson, I., and Booch, G. (2004): Unified Modeling Language Reference Manual, The $\left(2^{\text {nd }}\right.$ Edition). Pearson Higher Education. ISBN: 0321245628.

[13] Vara, J., Snchez, J., and Pastor, O. (2013): "On the Use of Goal Models and Business Process Models for Elicitation of System Requirements". English. In: Enterprise, Business-Process and Information Systems Modeling. Ed. by Selmin Nurcan, HenderikA. Proper, Pnina Soffer, John Krogstie, Rainer Schmidt, Terry Halpin, and Ilia Bider. Vol. 147. Lecture Notes in Business Information Processing. Springer Berlin Heidelberg, pp. 168-183. ISBN: 978-3-642- 38483-7. DOI: $10.1007 / 978-3-642-38484-4$ 13. URL: http: //dx.doi.org/10.1007/978-3-642-38484-4 13.

[14] Yu, E.S.K. (1997): “Towards modelling and reasoning support for early-phase requirements engineering". In: Requirements Engineering, 1997., Proceedings of the Third IEEE International Symposium on, pp. 226-235. DOI: 10.1109/ISRE. 1997.566873. 